\title{
Analysis of a Special Period Sampled Bragg Grating (PSBG) Structure Combined with the Large Chirp Parameter
}

\author{
Anurag Kumar Yadav \\ M.Tech scholar \\ Dept. of ECE \\ SHIATS, ALLAHABAD
}

\author{
A.K. Jaiswal \\ Professor and H.O.D \\ Dept. of ECE \\ SHIATS, ALLAHABAD
}

\author{
Neelesh Agrawal \\ Asst. Professor \\ Dept. of ECE \\ SHIATS, ALLAHABAD
}

\begin{abstract}
Chirped fiber Bragg grating (CFBG) provides an attractive solution for low cost dispersion compensation in a fiber optic transmission system. This kind of grating can be produced with a large chirp parameter and period sampled distribution along the grating length. The present work carries out the design optimization of a chirped FBG in respect of chirp bandwidth and apodization in achieving optimum dispersion compensation in a 20Gbps optical transmission link for different modulation formats.
\end{abstract}

\section{Keywords}

Fiber grating; Large chirp parameter; Period Sampled Distribution; Dispersion compensation; chirp bandwidth Grating Length, apodization optimum.

\section{INTRODUCTION}

Linearly chirped fiber Bragg gratings are commonly used for dispersion compensation in 10Gbps optical transmission systems. The availability of mass production techniques and low cost has made this device very attractive for dispersion compensation. Even in higher bit rates such as $20 \mathrm{Gbps}$ case they are also found to be very effective.

Chirped Fiber Bragg gratings (CFBGs) are utilized for achieving dynamic chromatic dispersion. This can be done by fabricating the grating such that the grating space varies linearly over the length of the fiber grating.

Incident spectrum

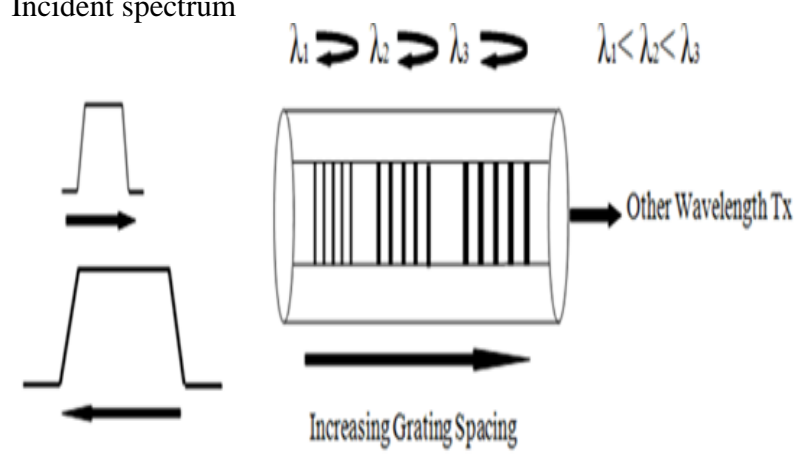

Reflected spectrum
This make the range of wavelength satisfy the Bragg condition for reflecting Fig1 shows that the grating spacing increases only the fibre which means that the Bragg wavelength increases with distance along the grating length. With this the longer wavelength output of a pulse travel farther into the fiber before being reflected. Hence they experience more delay in going though the grating the shorter wavelength cartent the relative delays introduced by the grating on the different wavelength component of the pulse are similar to the delays caused by the fiber in the anomalous region. Thus, we find that this type of chirped grating provides the boarding of the pulse. However, if the sequence of grating spacing is reversed (spacing decreases along the fiber), this will faring in effect of compression of the pulse there by causes the chromatic dispersion compensation.

\section{CHIRPED SAMPLED FIBER BRAGG GRATING}

In wide bandwidth, there are multiple channels and each channel has totally different dispersion character and Bandwidth. The grating period distribution can be expressed by

$$
\begin{aligned}
& \Lambda_{o}(z)=\Lambda_{c}-C_{g 1} z \quad\left(|z| \leq \frac{s p}{2}\right) \\
& \Lambda(z)=\Lambda_{o}(z-n s p) \quad\left(\frac{s p}{2}<|z| \leq \frac{l}{2}\right)
\end{aligned}
$$

Where $\mathrm{n}=\ldots \ldots \ldots,-2,-1,0,1,2,3,4,5 \ldots \ldots \ldots$

where $C_{g 1}$ is linear chirp parameter, $s p$ is the period sample length, $\mathrm{z}$ is the position coordinate which has zero point in the middle of the grating, 1 is the grating length, $\Lambda_{c}$ is the central period of the grating, $\Lambda_{0}(\mathrm{z})$ is the period distribution inthe middle sample of the grating, $\Lambda(z)$ is the period distribution on the other place of the grating. $n_{e f f}$ is the refractive index

$\delta n$ is modulation depth .

Following fig. 2 shows Period and index modulation distribution along the grating length with specified parameters 1. $s p, C_{g}, n_{e f f}, \delta n$ and $\Lambda_{c}$ of PSBG

Fig.1 Chirped Fiber Bragg gratings 

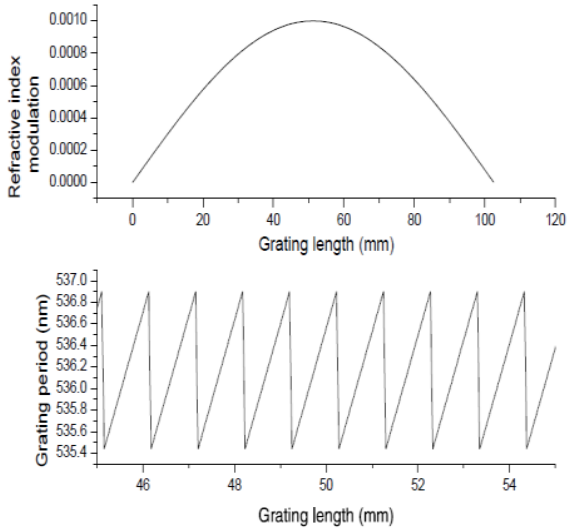

Fig. 2. Period and index modulation distribution alongthe grating length

\subsection{Performance of a chirped FBG}

The performance of a chirped FBG is controlled by several parameters such as (1) the length of the grating used for compensation, (2 ) chirp bandwidth,(3) apodisation profile,(4) ac index modulation depth (5) the number of uniform steps in the grating .

Several studies are carried out to optimize design procedure for dispersion compensating chirped FBG.

\subsection{Procedure for the optimized design of a chirped FBG as a Dispersion Compensator;}

Several parameter are to be considered which are related to the grating in chirped FBG used as dispersion compensator these are(1) grating length,(2) chirped bandwidth and (3) apodisation profile.

Conventional approach for choosing the length of the grating (Lg) is based on the knowledge of the signal bandwidth and amount of the fiber dispersion to be compensated. Such a procedure needs adhoc trial of fixing the length of the grating to be $2 \mathbf{L g}, 3 \mathbf{L g}$ etc in order to achieve the best performance.

One of the approach for design optimization is to the chose of the FBG length based on appropriate selection of chirped bandwidth containing a desired level of signal energy threshold $\mathrm{E}_{\text {th }}$ depending on the performance optimization required.

It is known that optimization of apodisation profile has a distinctive role in ensuring minimum delay ripple across the grating bandwidth

Symmetric and asymmetric tanh apodisation are used to achive design optimization

The design of chirped FBG for dispersion compensation requires proper choice of various grating related parameters. The following steps are involved in the design.

\section{1) Calculation of grating length as}

$$
L_{g}=L_{f} B D\left(\lambda^{2} / 2 n\right)
$$

$\boldsymbol{L}_{\boldsymbol{f}}$ is the length of the fiber, $\mathbf{D}$ is the fiber dispersion parameter $(\mathrm{ps} / \mathrm{nm} / \mathrm{km}), \mathbf{B}$ is the signal bandwidth, $\boldsymbol{\lambda}$ is the central wavelength and $\mathbf{n}$ is the effective refractive index.

2) Number of Uniform sections:

For a given length $\mathrm{Lg}$ the number of uniform sections is chosen to be $\mathbf{8 0}$ or higher. The grating simulation is carried out by transfer matrix approach for an adequately chosen value of the chirp bandwidth, neff $=\mathbf{1 . 4 5}$, ac modulation index at optimized value $1.2 \times 10-4$.

3) Chirped Bandwidth:

Chirped bandwidth is chosen so as to optimize the grating length to be $\mathbf{2} \mathbf{L g}, \mathbf{3} \mathbf{L g}$ etc. in order to achieve the optimum result. It may be noted that the above choice of length also varies depending on the signal modulation used. This may referring to Table 1 which shows that for the Eye opening penalty (EOP) of $\mathbf{0 . 2} \mathrm{dB}$ for the dispersion compensated 20 Gbps signal at the output of the grating, the grating length differs depending on the value of the chirp bandwidth and the modulation format. However the design procedure adopted as descended in pares $12 \& 3$ is not appropriate because the detarled nature of signal spectrum has not coverdered.

4) proposed design approach

In this design we tried to optimize the design in terms of the length of the grating, chirp bandwidth and apodisation. For all other parameters standard values are adopted. DC index modulation of zero and AC index modulation of 1.2x10 -4 are taken for all the cases. Number of steps in the calculation of grating is chosen to be $\mathbf{1 0 0 .}$

5) Performance characteristics of the system adopted $Q$ factor and Eye Opening Penalty (EOP)are the two components for the performance if grating based system. Eye-pattern is achieved at the receiver-end and $Q$-factor of the received signal is measured.

a) The $Q$ factor represents the statistical variation in the received signal and is defined by

$$
Q=\frac{\left|M_{o}-M_{1}\right|}{\sigma_{o}+\sigma_{1}}
$$

where, $M_{0}$ and $M_{1}$ are the mean and $\sigma_{0}$ and $\sigma_{1}$ are the standard deviation of the received signal measured for binary data" 0 " and "1", respectively.

b) Eye Opening Penalty (EOP) is defined as the ratio of eye height of the single channel to that of multiple channels and is measured in decibel. It can be expressed as

$$
E O P(d b)=10 \log \left(E O s_{C} / E O m_{c}\right)
$$

where $\mathrm{EOs}_{\mathrm{c}}$ and $\mathrm{EOm}_{c}$ are the eye-opening height of the single channel and multiple channels, respectively. Different system parameters like launch power, distance, channel count and channel spacing are varied to observe the impact of nonlinear effects on system performance.

\section{SIMULATIONS SETUP}

We have used the parameters as shown in Table 1 to design a PSBG and use its -2 order channel in the system dispersion compensation. The dispersion system model is shown in Fig. 2.The DFB laser frequency is chosen at $1553.1 \mathrm{~nm}$. The output power of the DFB is $1 \mathrm{mw}$. Continuous wave (CW) light is externally modulated at $10 \mathrm{Gbits} / \mathrm{s}$ with an NRZ pseudorandom binary sequence in a chirp less MachZehnder modulator having a $30 \mathrm{~dB}$ extinction ratio. The signal is launched into a link consisting of dispersion 
compensation normalized section with PSBG. The fiber loss is compensated by Erbium-doped optical fiber amplifier (EDFA). The calculation of the propagation in the optical fibers is performed using a standard split-step algorithm with adaptive step size. The EDFA is modeled by wavelength independent gain and the noise is ignored for only concentrating on the influence of dispersion and nonlinear effects in the transmission system. The draw the eye diagram of the signal propagating at $\mathrm{M}-\mathrm{Z}$ modulator, through fiber length and through the PSBG.

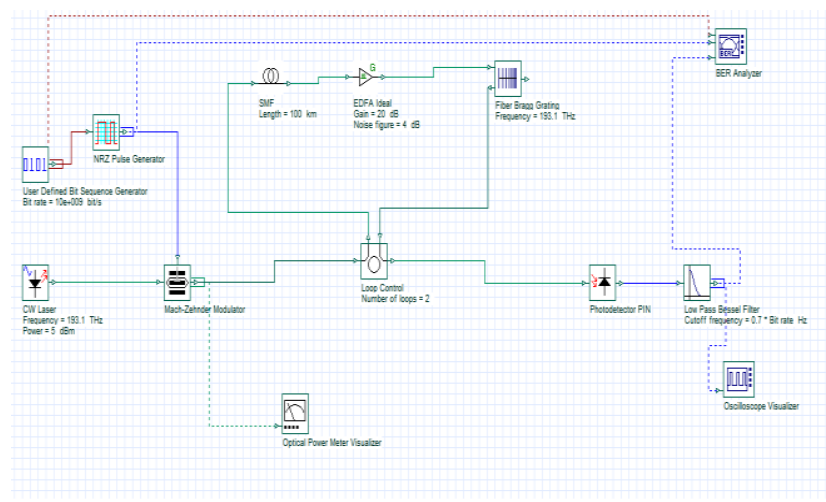

Fig.3 Simulation setup FBG

Table 1. Fiber Parameters

\begin{tabular}{|c|c|}
\hline Dispersion $(\mathrm{ps} / \mathrm{nm} / \mathrm{km})$ & 17.0 \\
\hline $\begin{array}{c}\text { Dispersion slope } \\
(\mathrm{ps} / \mathrm{nm} / / \mathrm{km})\end{array}$ & 0.050 \\
\hline $\begin{array}{c}\text { Nonlinear coefficient } \\
(\mathrm{mW} / \mathrm{km})\end{array}$ & 0.0012 \\
\hline Loss coefficient $(\mathrm{dB} / \mathrm{km})$ & 0.20 \\
\hline Power $(\mathrm{dB})$ & 5.0 \\
\hline
\end{tabular}

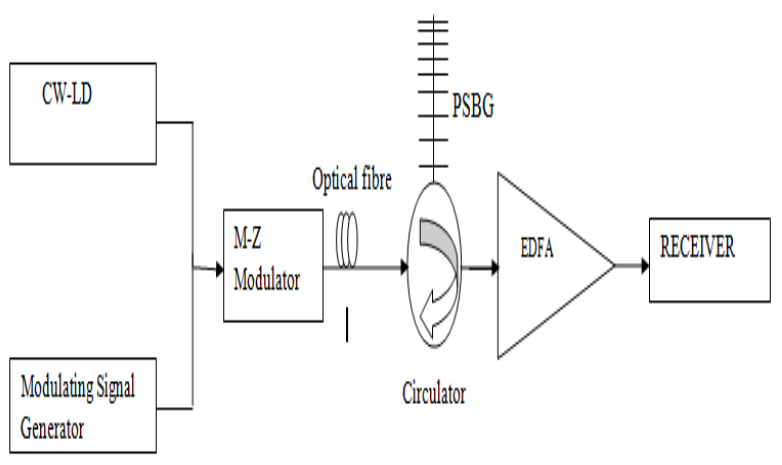

Fig.4 Dispersion compensation system model

\section{RESULTS AND DISCUSSION}

The simulation and optimization of the design is done by OptiSystem 13.0 simulation software. The eye opening penalty (EOP)(dB), MIN BER, $\mathbf{Q}$ factor diagrams and results of output power, Signal power $(\mathbf{d B m})$, are indicate the table using different values of length of fiber $(\mathrm{km})$, and variable length of FBG ( $\mathbf{m m})$. The design optimization of chirped FBG as dispersion compensator is presented.
It has been observed that While fiber length $(\mathrm{km})$ is increasing. The EOP $(\mathrm{dB})$ is decrease during the interval of ( 5 to 28.75$) \mathrm{Km}$ then starts increasing and Q FACTER increases to the max point and then starts decreasing, while in the case of BER, initially it starts with a negligible change for some $\mathrm{km}$ then starts rising speedily. While dispersion is increase the EOP $(\mathrm{dB})$ is increases.

While increasing the chirp length, the EOP $(\mathrm{dB})$ decreases and Q FACTER start increasing to the maximum point then decreases to the zero. At the same time BER getting decreases till the chirp length of $2 \mathrm{~cm}$ then remains constant for the chirp length between $2 \mathrm{~cm}$ to $4 \mathrm{~cm} \&$ then increases rapidly till the chirp length of $5 \mathrm{~cm}$. and eye diagram shown in graph, Q Factor and eye height.

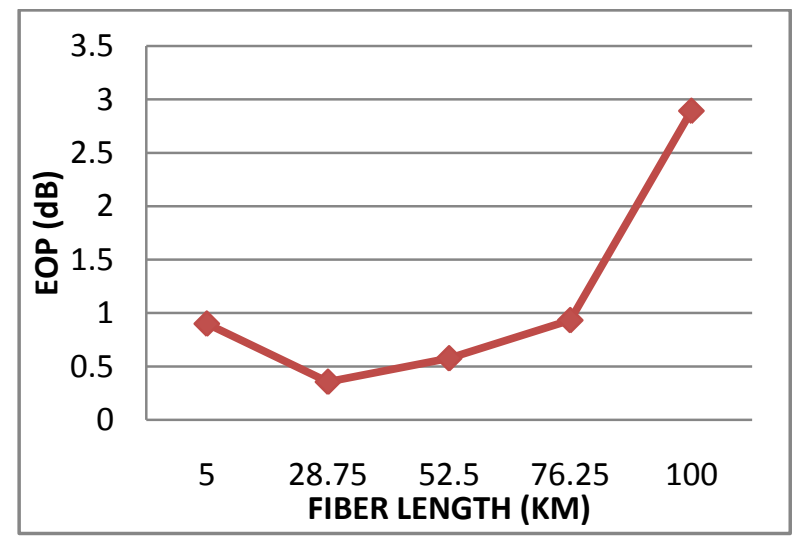

Fig 5.1 EOP (dB) Vs Length (KM)

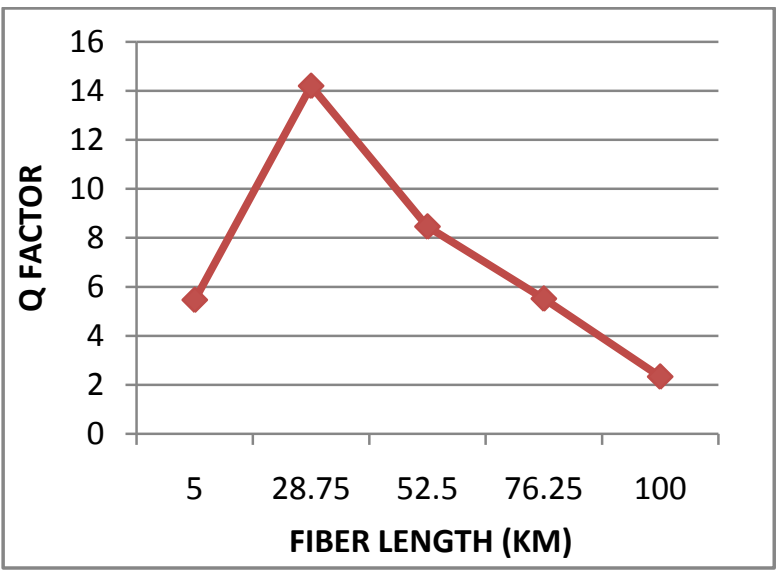

Fig 5.2 Q factor Vs length $(\mathrm{km})$

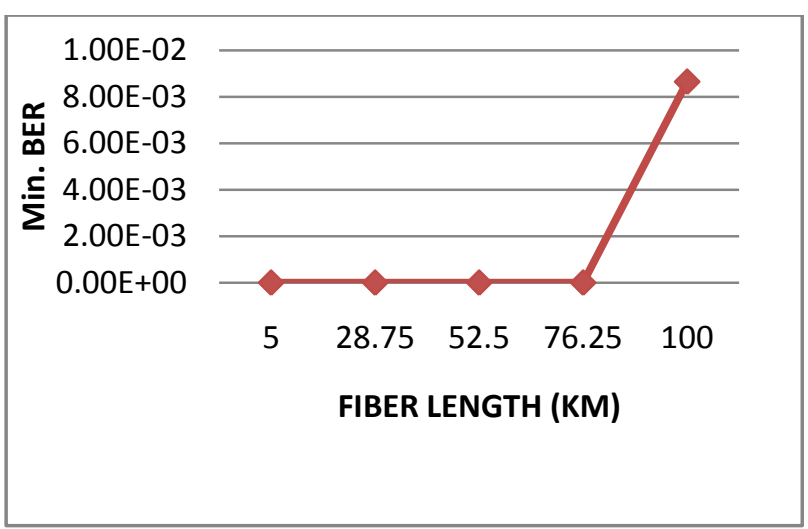


Fig 5.3 Min. BER Vs Length (km)

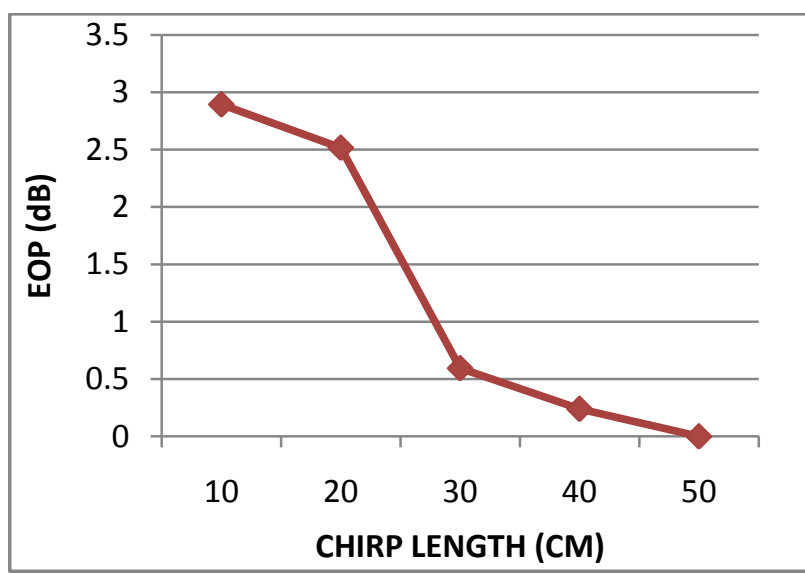

Fig 5.4 EOP (dB) Vs Chirp Length

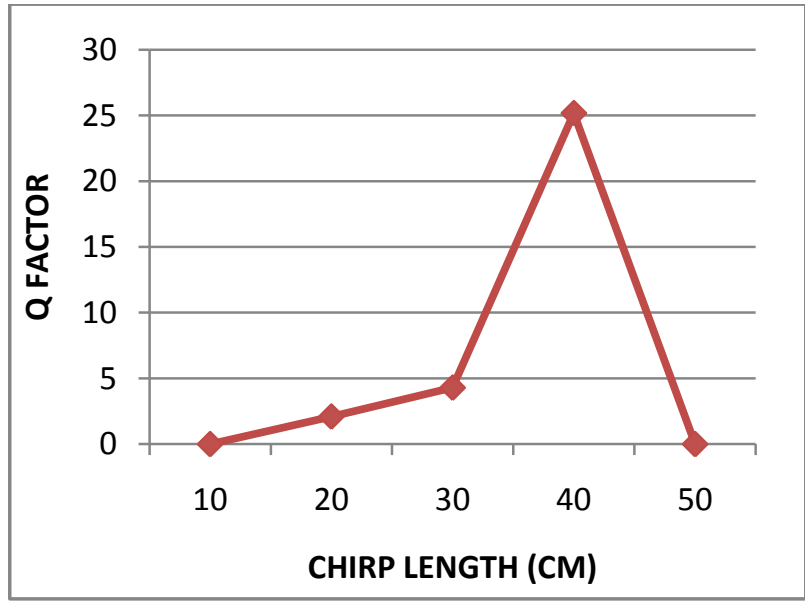

Fig 5.5 Q Factor Vs Chirp Length

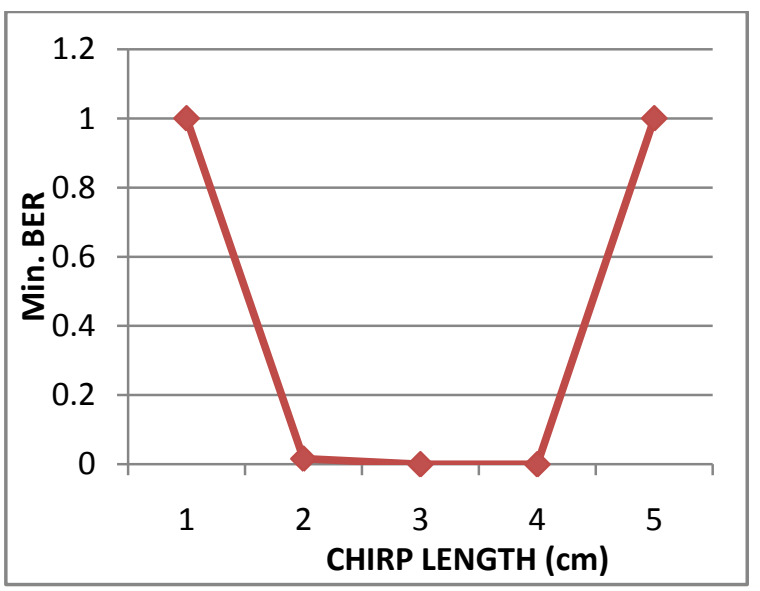

Fig 5.6 Min BER Vs Chirp Length

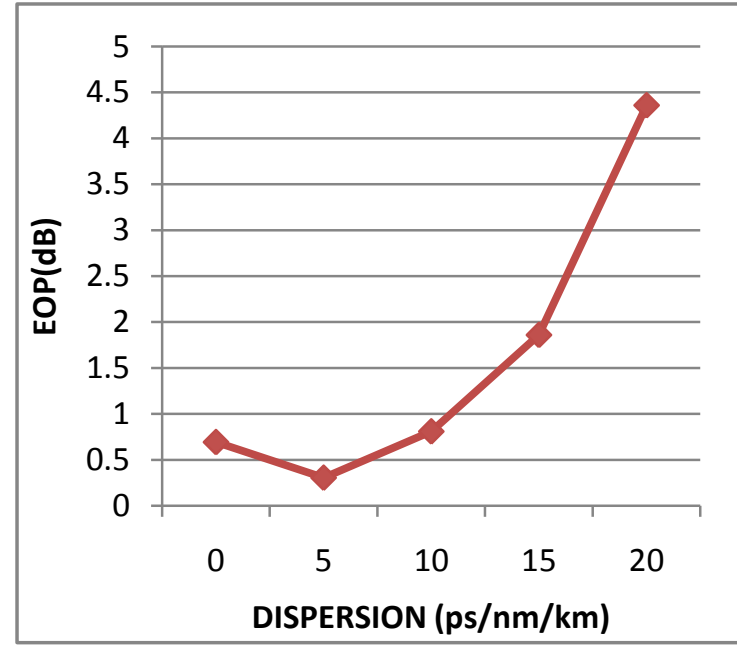

Fig 5.7 EOP Vs DISPERSION

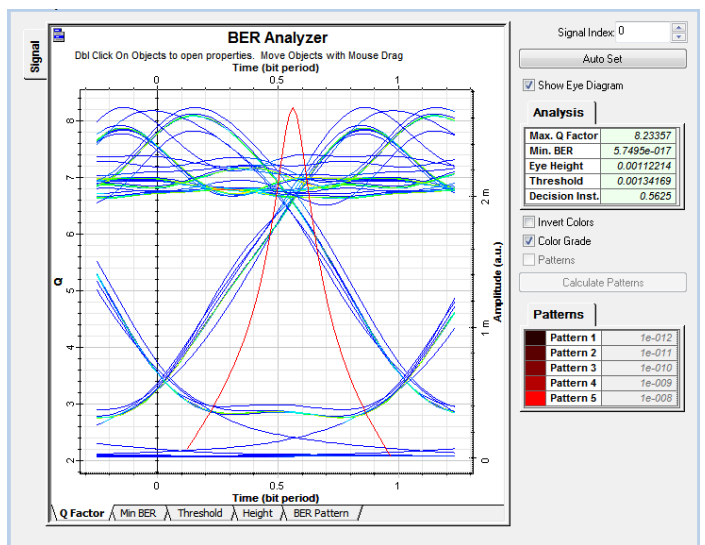

Fig. 5.8 EYE DIAGRAM

\section{CONCLUSION}

A simple design optimization of chirped FBG as dispersion compensator is presented. The criterion for appropriate choice of the chirp length $(\mathrm{cm})$ based on threshold energy of the signal allows the determination of grating length automatically optimized.

The performance result of simulation based design of chirped grating(SPFG) for dispersion compensation is expressed in terms of EOP Vs chirp length and also EOP Vs Dispersion . Appropriate chirp length needs to be decided in the graph for obtaining the required EOP.

\section{ACKNOWLEDGEMENT}

I would like to thank my adviser Prof. A. K. Jaiswal for his support and encouragement. I would also like to thank Asst Prof. Neelesh Agrawal and Asst. Prof. Mukesh Kumar to

provide valuable advices. The product of this research paper would not be possible without all of them.

\section{REFERENCES}

[1] Phing, H. S., Ali, J., Rahman, R. A., Tahir, B., A., (2012). "Fiber Bragg grating modeling, simulation and characteristics with different gating length," Journal of Fundamental Sciences 3, 1612-1125. 
[2] Navneet Singh Aulakh, (2010) " Investigations on fiber bragg gratings for fiber optic communication systems, " , department of electronics \& communication engineering thapar university.

[3] Kenneth O. Hill and Gerald Meltz, (1997)"Fiber Bragg Grating Technology Fundamentals and Overview", Journal of Lightwave Technology, vol 15, no. 8, pp. $1263-1274$.

[4] Xu M.G.; Geiger H.; Archambault, J.L.; Reekie, L.; Dakin, J.P.,(1993) "Novel interrogating system for Fiber Bragg Grating sensors using an acousto-optic tunable filter," Electronics Letters , vol.29, no.17, pp.15101511.

[5] Ferreira L.A.; Diatzikis E.V.; Santos J. L.; Farahi F., (1998) "Frequency-modulated multimode laser diode for Fiber Bragg Grating sensors," Lightwave Technology, Journal of , vol.16, no.9, pp.1620-1630.

[6] Murphy D.F.; Flavin D.A.; McBride, R.; Jones, J.D.C., (2002) "Spatially scanned interferometric interrogation of Fiber Bragg Grating sensors based on Hilbert transform processing," Optical Fiber Sensors Conference Technical Digest, 2002. OFS 2002, 15th , vol., no., pp. 379-382 vol.1

[7] Teunissen, J.; Merte, R.; Peier, D., (2002) "Stability of Fiber Bragg Grating sensors for integration into highvoltage transformers for online monitoring," Optical Fiber Sensors Conference Technical Digest, 2002. OFS 2002, 15th , vol., no., pp. 541-544 vol.1.

[8] Fernandez A.F.; Brichard B.; Borgermans P.; Berghmans F.; Decreton M.; Megret P.; Blondel, M.;
Delchambre, A., "Fiber Bragg Grating temperature 190 sensors for harsh nuclear environments, (2002)" Optical Fiber Sensors Conference Technical Digest, 2002. OFS 2002, 15th , vol., no., pp. 63-66 vol.1.

[9] Betz, D.; Staudigel, L.; Trutzel, M.N., "Test of a Fiber Bragg Grating sensor network for commercial aircraft structures,(2002)" Optical Fiber Sensors Conference Technical Digest, 2002. OFS 2002, 15th , vol., no., pp. $55-58$ vol. 1 .

[10] Hua Lu; Hussain, R.; Ming Zhou; Xijia Gu, (2009) "Fiber Bragg Grating Sensors for Failure Detection of Flip Chip Ball Grid Array in Four-Point Bend Tests," Sensors Journal, IEEE, vol.9, no.4, pp.457-463.

[11] Ping Lu; Liqiu Men; Qiying Chen, (2009) "PolymerCoated Fiber Bragg Grating Sensors for Simultaneous Monitoring of Soluble Analytes and Temperature," Sensors Journal, IEEE , vol.9, no.4, pp.340-345.

[12] M.A. Othman1, M.M. Ismail2, H.A. Sulaiman3, M.H Misran4, M.A. Meor Said5, Y.A. Rahim6, A.N. Che Pee12, M.R. Motsidi, (2012) "An Analysis of 10 Gbits/s Optical Transmission System using Fiber Bragg Grating (FBG) ,’IOSR Journal of Engineering (IOSRJEN) ISSN: 2250-3021 Volume 2, Issue 12

[13] E. Gemzický a, J. Müllerová (2009) Numerical analysis of reflection characteristics of cascaded non-uniform fiber Bragg gratings," Department of Engineering Fundamentals, Faculty of Electrical Engineering, University of Žilina, Optical Sensors, Proc. of SPIE Vol. 12356, 123561R 